\title{
Zola et Rolland : deux paradigmes du travail génétique
}

\section{Céline Grenaud-Tostain}

\section{(2) OpenEdition}

1 Journals

Édition électronique

URL : https://journals.openedition.org/genesis/1637

DOI : 10.4000/genesis. 1637

ISSN : 2268-1590

Éditeur :

Presses universitaires de Paris Sorbonne (PUPS), Société internationale de génétique artistique littéraire et scientifique (SIGALES)

Édition imprimée

Date de publication : 9 mai 2016

Pagination : 87-95

ISBN : 9791023105315

ISSN : 1167-5101

\section{Référence électronique}

Céline Grenaud-Tostain, «Zola et Rolland : deux paradigmes du travail génétique », Genesis [En ligne], 42 | 2016, mis en ligne le 05 juin 2017, consulté le 17 janvier 2023. URL : http://

journals.openedition.org/genesis/1637 ; DOI : https://doi.org/10.4000/genesis.1637

Tous droits réservés 


\title{
Zola et Rolland : deux paradigmes du travail génétique
}

\author{
Céline Grenaud-Tostain
}

L a confrontation de deux modèles d'écriture aussi différents que ceux dont sont issues les œuvres d'Émile Zola (1840-1902) et Romain Rolland (1866-1944) doit faire surgir un surplus de signification au niveau génétique. Elle permet de s'interroger sur les modalités d'intégration du réel vécu à la fiction pensée, autrement dit de mesurer l'impact, sur le travail des deux écrivains, des événements qui ont jalonné leur existence et, peut-être, modifié la trajectoire des romans en gestation. Comment les cycles des Rougon-Macquart, des Trois Villes et des Évangiles d'une part, de Jean-Christophe et de L'Âme enchantée d'autre part ont-ils été façonnés et refaçonnés par les accidents, heureux ou malheureux, qui ont bouleversé les deux auteurs, parfois remis en cause les plans tirés sur la comète prérédactionnelle? Quelle part l'intime a-t-il pu prendre dans l'acte de création et, publication oblige, dans celui d'exposition de soi ? Ces questions feront saillir deux tendances et même, tant les réponses proposées par Zola et Rolland diffèrent sur ce point, deux paradigmes génétiques. D'un côté, l'écriture est appréhendée comme un acte relativement indépendant de son créateur et des émois qui le secouent, à tel point qu'au plus fort de la tourmente, elle peut faire office d'échappatoire et, selon la célèbre formule d'une lettre de $1885^{1}$, de formidable tremplin vers les étoiles. De l'autre, l'art et l'existence sont intimement mêlés, tant et si bien que les éléments tricotés dans la sphère personnelle se nouent à ceux tissés dans l'atelier de création. Interférence qui fait signe vers le pacte autobiographique sans toutefois le signer tout à fait, puisque l'œuvre persévère à être un produit de l'imagination et ne s'offre à lire sous l'éclairage de la catharsis ou de la biographie fictionnelle qu'à l'issue d'un décryptage aussi précis que celui mené par Bernard Duchatelet pour Jean-Christophe ${ }^{2}$. Cela dit, la distinction de ces deux paradigmes n'est pas toujours d'une netteté suffisamment tranchée pour interdire les croisements. Il arrive bien sûr que Zola laisse une part de lui-même infléchir le projet esquissé dans les dossiers préparatoires ou, inversement, que Rolland passe sous silence un élément de sa propre biographie qu'il aurait pu facilement intégrer à la trame de ses récits. Chacune des grilles de lecture devra donc paradoxalement servir à l'analyse de l'œuvre pour laquelle elle semblait a priori la moins appropriée, renversement qui pourra révéler la complémentarité des deux approches, au lieu de vouer celles-ci à la stricte opposition.

\section{Les enjeux}

L'étude que nous nous proposons de mener a pour premier enjeu le repérage de l'instance auctoriale au sein des textes programmatiques. On peut en effet s'interroger sur les modalités qui permettent à la figure de l'auteur, en l'occurrence au cours de la genèse d'un cycle, de dépasser les critères de base qui la fondent, tels qu'ils sont habituellement reconnus depuis Foucault ${ }^{3}$. Au-delà de l'appropriation d'un texte, ici d'un avant-texte donc, et de son attribution, de la valeur littéraire d'un discours, de son style et de son statut, du contexte historique à décoder; au-delà, donc, de ces critères plutôt désacralisants, mais quelque peu déshumanisants voués à définir l'auteur, on est en droit de se demander comment le sujet créateur parvient à parler de lui, quand bien même il semble uniquement pointer le récit qu'il projette d'écrire. Au-delà de l'auteur, il faudrait retrouver l'homme, au-delà de l'œuvre pour autrui le discours sur soi, au-delà de la fiction le vécu. Ce faisant, il importe d'éviter deux

1. Émile Zola, Lettre à Henry Céard du 22 mars 1885, dans Correspondance, Montréal / Paris, Presses de l'Université de Montréal / Éditions du CNRS, t. V, 1985, p. 249 : «J'ai l'hypertrophie du détail vrai, le saut dans les étoiles sur le tremplin de l'observation exacte. »

2. Bernard Duchatelet, La Genèse de Jean-Christophe de Romain Rolland, Paris, Minard, 1978.

3. Michel Foucault, «Qu'est-ce qu'un auteur?», Bulletin de la Société française de philosophie, 63e année, $\mathrm{n}^{\circ} 3$, juillet-septembre 1969 , p. $73-104$ 
écueils. Il ne saurait être question ni de substituer à la fiction en gestation la notion de roman autobiographique - point de confusion des genres -, ni de se perdre dans un dépistage exhaustif de l'ethos auctorial. Car au fond, il serait tentant de vouloir multiplier presque à l'infini les ponts entre le sujet et son objet de création. Mais pour valider la pertinence des deux paradigmes, il faudra s'en tenir aux liens les plus solides et les plus révélateurs.

En outre, l'émergence de deux paradigmes de la création permet, puisqu'elle est étroitement liée à la présence-absence du sujet écrivant, de revenir sur l'engagement des deux hommes. Ce n'est pas le moindre des paradoxes, ou plutôt des chiasmes, que celui au détour duquel on découvre l'intellectuel engagé par excellence se mettre généralement en retrait dans sa propre fiction et, au contraire, l'auteur aspirant à l'indépendance de l'Esprit ${ }^{4}$ laisser sa propre existence dicter le remaniement de ses romans. Dès lors, c'est la notion même d'engagement qui tend à être redéfinie, du moins complétée, à la lumière d'une philosophie aux accents kierkegaardiens. Zola et Rolland sont surpris à la fois au stade éthique, dans le respect de valeurs dont résulte directement leur travail et, au stade religieux, dans une solitude qui les oblige à confronter leur foi, au sens laiic du terme, à l'absurdité dont tend à se couvrir le réel. Deux questions découlent de cette redéfinition. Primo, comment les valeurs personnelles et sociales nourries par chacun ont-elles été capables d'infléchir le projet esthétique? Secundo, comment les auteurs ont-ils intégré à leur écriture l'expérience plus ou moins douloureuse de l'exil ou celle, extrêmement déstabilisante, d'une transcendance en butte avec le néant? En somme, il s'agit de pointer les conséquences sur l'écriture de l'engagement audacieux de son propre vécu ou, à l'inverse, d'un désengagement qu'il est préférable de désigner, de manière plus constructive, comme repli salvateur de soi loin de soi.

Dernier intérêt de notre analyse, nous semble-t-il, celui qui réside dans la comparaison de deux écrivains qu'on pense trop rarement à rapprocher. La silhouette de Zola, chef de file du naturalisme et figure emblématique du dreyfusisme, vient pourtant aisément s'aligner aux côtés de celle de Rolland. Qu'on mesure la remarquable faculté de ce dernier à gérer l'héritage réaliste de l'écriture cyclique, sans pour autant verser dans la répétition surannée d'un modèle ayant déjà atteint sa perfection ${ }^{5}$. Qu'on ajoute aux considérations strictement littéraires la représentativité du Prix Nobel 1915, sur le plan idéologique et intellectuel, au regard d'une valeur menacée d'effritement en 1914, comme l'a été un autre pilier des civilisations dans les années 1890. Le pacifisme vaut bien, mutatis mutandis, la pierre angulaire du combat dreyfusard, cette Justice souveraine inscrite au cœur du credo zolien et jusque dans le dernier Évangile programmé avant la mort. La presque évidence de la comparaison a été gommée, sans doute, par la tendance, encore très nette de nos jours, à délaisser l'œuvre de Rolland, en particulier sa production romanesque, et à considérer celui qui marqua pourtant toute une génération comme un auteur plus ou moins mineur. Contribuons donc à remédier à ce relatif gommage.

\section{Dépasser l'intime}

Désormais que les enjeux sont posés, il convient d'en venir aux deux paradigmes que nous placerons pour l'un sous le signe du dépassement (de soi, de son vécu), pour l'autre sous celui de l'intrication (la création pouvant dès lors être envisagée comme un mode de résilience).

L'écriture de Zola, régie dès 1864 par la théorie des écrans, suppose l'existence métaphorique d' «un simple verre à vitre [...] parfaitement transparent», permettant à l'artiste de parvenir «à un haut degré de reproduction exacte $^{6}$ ». En ce sens, tous les éléments trop directement biographiques interfèrent comme des obstacles faisant barrage à l'idéal naturaliste et tendent à être évacués par l'écrivain. Les dossiers préparatoires sont beaucoup plus les dépositaires d'un projet et d'un travail que ceux de confessions tourmentées. Empruntons, pour les relire, les catégories de la rhétorique classique déjà exploitées par les chercheurs zoliens dans Le Signe et la consigne, sous

4. «La Déclaration d'indépendance de l'Esprit », publiée dans L'Humanité du 26 juin 1919, a été cosignée par de nombreux intellectuels, «Travailleurs de l'Esprit» du monde entier unis autour de la vérité.

5. De même que Balzac chez Zola, Tolstoï constitue un contre-modèle à dépasser chez Rolland, par ailleurs nourri de lectures réalistes (Dickens, Daudet, Tourgueniev, Dostoïevski...).

6. Émile Zola, lettre à Antony Valabrègue du 18 août 1864, dans Correspondance, éd. Alain Pagès, Paris, Garnier-Flammarion, 2012, p. 116. 
la direction de Philippe Hamon 7 . On est d'abord tenté de s'en remettre à la memoria pour retrouver la trace du sujet pensant. L'intratextualité (avec les souvenirs procédant des Rougon-Macquart antérieurs à celui en cours) et l'intertextualité (comprise entre tentation de l'imitation et désir de dissociation) donnent lieu à des analyses très riches. En revanche, les références autobiographiques sont en définitive extrêmement rares et ne permettent pas d'étoffer la compréhension du cogito, comme le souligne Chantal Pierre en établissant, à cet égard, une distinction avec les manuscrits du plus égotiste Stendhal 8 . Ces références, quand elles sont explicites, se limitent généralement au renvoi à des modèles assumés : les amis des années de jeunesse pour L'Euvre, le père de Cézanne pour La Conquête de Plassans, etc. C'est dire que le romancier, jusque dans les textes programmatiques, s'efforce sinon de ligoter, du moins d'ignorer ses propres affects et les souvenirs trop marquants qui pourraient influencer son écriture.

Ce silence de l'auteur sur lui-même autorise qu'on recherche le surgissement de l'intime à d'autres niveaux des avant-textes, à commencer par l'elocutio. Le soin accordé par Zola au choix des mots, jusque dans les fiches-personnages ou les folios consacrés au plan, apparaît, sur le plan strictement graphique, à la faveur des guillemets, soulignements et, même si elles ne sont pas nombreuses, des ratures et biffures. Quand les remords font place au lapsus, ils invitent à une lecture plus intime des dossiers préparatoires. La confusion entre «scène» et «Seine», dans celui de L'Euvre ${ }^{9}$, encourage ainsi à ancrer la production zolienne dans le microcosme de la capitale, quand bien même les personnages du roman en cours sont paradoxalement inspirés de connaissances aixoises. Mais ce genre de lapsus n'est pas fréquent. Les textes programmatiques sont dotés d'une grande clarté et contiennent, on le sait, relativement peu de coquilles ou de corrections, ce que l'on peut traduire de deux façons. On peut y lire une forme d'indifférence vis-à-vis de l'acte manqué lexical et comme un refus délibéré de relire l'avant-texte pour le modifier encore et encore. C'est reconnaître dans ce cas la volonté, chez Zola, de ne point accorder une place trop grande à l'instance qui régit, consciemment ou non, le choix du vocabulaire et les éventuelles fautes commises çà et là. Mais la résistance opposée à la reprise du dossier peut aussi être interprétée comme une forme de lâcher-prise. Zola ne craint visiblement pas de laisser quelque erreur trahir son état d'esprit ou même ses considérations à l'égard d'un sujet qui le concerne (Aix-en-Provence pour les quatre RougonMacquart qui se déroulent à Plassans ${ }^{10}$, le théâtre dans Nana, la création dans L'Euvre...). En somme, l'elocutio fait surgir de nouveaux paradoxes plutôt qu'elle n'en résout et c'est encore vers une autre catégorie qu'il faut se tourner pour espérer accéder au sujet.

Grâce à l'inventio, peut-être sera-t-il permis de surprendre l'auteur tel qu'en lui-même, privilégiant l'élection de thèmes récurrents et s'autorisant l'élaboration d'une fantasmagorie privée. Cet aspect-là d'une œuvre est plutôt délicat à traiter, car difficile à circonscrire : où poser la limite de l'inspiration strictement personnelle? Ici, deux exemples bien connus viennent à l'esprit. On évoquera volontiers le cas de La Joie de vivre (1884), programmé dès 1880 sous le titre de Pauline Quenu11 et rédigé du 25 avril au 23 novembre 1883. La crise morale éprouvée dans les années 1880 - avec les disparitions successives des amis Edmond Duranty (le 9 avril, dans la misère) et Gustave Flaubert (le 8 mai, dans le coup de foudre d'une hémorragie cérébrale), puis celle d'Émilie, la mère tant aimée (le 17 octobre) - est le terrain de désolation sur lequel a pu facilement se développer l'étude de la philosophie schopenhauerienne. A fortiori si l'on observe que La Joie de vivre ne faisait pas partie des dix romans programmés dans le plan originel de 1868, on est en droit de tirer un trait d'union entre le réel et la fiction. Dans le même registre, Le Docteur Pascal (1893) doit beaucoup à la seconde jeunesse vécue par l'écrivain aux côtés de Jeanne Rozerot. La scène finale permet au cycle, contre toute attente, de s'achever dans un relatif optimisme. L'image de «l'enfant, qui tétait toujours, son petit bras en l'air, tout droit, dressé comme un drapeau d'appel à la vie ${ }^{12} \gg$ fait

7. Le Signe et la consigne. Essai sur la genèse de l'œuvre en régime naturaliste, Zola, dir. Philippe Hamon, Genève, Droz, 2009.

8. Ibid., p. 197.

9. Ibid., lapsus relevé par Philippe Hamon, op. cit., p. 212

10. La Fortune des Rougon (1871), La Conquête de Plassans (1874), La Faute de l'abbé Mouret (1875) et Le Docteur Pascal (1893).

11. C'est le 15 avril 1880, au journaliste du Réveil Fernand Xau, que Zola confie avoir neuf autres projets en tête, dont Pauline Quenu, pour compléter son cycle.

12. Émile Zola, Le Docteur Pascal, dans Euvres complètes, La Clôture. 1892-1893, t. XV, dir. Henri Mitterand, présentation par Jean-Sébastien Macke, Paris, Nouveau Monde Éditions, 2007, p. 573. 
écho au bonheur de la paternité éprouvé par Zola lui-même à la même époque. Le vécu éclaircit donc le tableau narratif ou, comme précédemment, l'assombrit; en tout cas, il n'est pas complètement désolidarisé du projet d'écriture. On ne peut parler d'adaptation, mais plutôt de la capacité du réel à donner le la. En imposant sa propre tonalité, le vécu parvient à infléchir le sens même du cycle, c'est-à-dire non seulement à l'aimanter vers un pôle négatif ou positif, mais aussi à lui conférer une nouvelle signification.

Cela dit, si déterminants ces exemples soient-ils, ils sont assez rares dans l'œuvre zolienne, par ailleurs dominée par une opiniâtreté dans le détachement à ce point remarquable qu'elle confine parfois au sacrifice. Nous choisirons comme cas le plus représentatif Fécondité (1899), pour lequel les Lettres à Alexandrine récemment éditées 13 constituent un précieux complément au matériel génétique (le volumineux dossier préparatoire ${ }^{14}$, les Pages d'exil15 et les quatre cent trente-sept lettres de la correspondance générale couvrant la période 1897-189916). Les lettres de Zola à sa femme, envoyées d'Angleterre durant l'année d'exil à raison d'environ deux par semaine, permettent de suivre la rédaction du premier Évangile et, en comblant l'absence de brouillons, de baliser très précisément le terrain de l'écriture : «J'avais commencé le roman, seul, le 4 août 98 , et viens de le terminer, seul, le 27 mai 9917.» Tantôt, elles donnent à voir l'auteur exaspéré par l'ostracisme auquel le condamne l'affaire Dreyfus - le dégoût de tout inspire cette pensée radicale selon laquelle «il vaudrait mieux la mort 18 »- et désespérant de pouvoir échapper à ses propres démons - «Je me suis levé la tête lourde, je n'ai pu écrire que trois pages ${ }^{19}$ ». Tantôt, les confidences à l'épouse permettent de brosser le portrait d'un homme apaisé par l'utopie évangélique dans laquelle il trouve refuge, rasséréné par l'acte de création - «Tout va bien, quand mon travail marche 20 » - et même gagné par le bonheur - «je ne suis jamais si heureux que lorsqu'on me laisse dans mon coin, à mon travail, à mes lectures et à mes réflexions 21 ». En dernier ressort, il apparaît que les souffrances endurées dans la retraite londonienne ont pour pendant une écriture au caractère lénitif et euphorisant, si bien qu'en 1901, Zola se retrouve à confier à Alexandrine : «J'en arrive parfois à regretter la grande solitude où je me trouvais en Angleterre ${ }^{22}$. » Cette nostalgie inattendue conduit à émettre deux hypothèses relativement au paradigme génétique du dépassement. D'une part, la rédaction, le travail sont assujettis à une logique du surpassement, de l'effort et presque de l'épreuve à la fois mentale et physique. Mais d'autre part, le dépassement vise un nirvana de la création entendu au sens d'une absorption totale et jouissive de l'individu dans l'universalité de son œuvre, par-delà ses propres désirs et angoisses. L'écriture se retrouve donc sublimée entre la nécessité de l'endurance et l'idéal d'un abandon absolu, exaltant, de soi pour autrui. Autant dire que cette abnégation est une forme d'ascétisme dont la régularité dans le travail - nulla dies sine linea, pas même les jours les plus sombres de l'Affaire - n'est rien moins que l'une des manifestations.

\section{L'intrication}

Aux antipodes de ce modèle se trouvent les avant-textes de Romain Rolland. Leur étude aboutit au constat d'une intrication beaucoup plus marquée entre le vécu et la fiction. La genèse de Jean-Christophe fait remonter à une note déterminante du 20 mars 1903 à l'occasion de laquelle l'auteur déclare «commence[r] d'écrire définitivement [s]on roman» et bâtit un plan général avec «cinq cahiers Péguy » composés chacun de deux parties 23 (la publication est prévue dans les Cahiers de la Quinzaine fondés en 1900). Mais pour compléter cette première donne génétique,

13. Émile Zola, Lettres à Alexandrine, dir. Alain Pagès et Brigitte ÉmileZola, avec la participation de Céline Grenaud-Tostain, Sophie Guermès, Jean-Sébastien Macke et Jean-Michel Pottier, Paris, Gallimard, 2014. 14. Dossier préparatoire de Fécondité, BnF, NAF 10348-10349.

15. Émile Zola, Pages d'exil, dans Euvres complètes, De l'Affaire aux Quatre Évangiles. 1897-1901, t. XVIII, dir. Henri Mitterand, présentation par Alain Pagès, Paris, Nouveau Monde Éditions, 2008, p. 539-558.

16. Émile Zola, Correspondance, t. IX, «L'affaire Dreyfus. 1897-1899», annotations par Owen Morgan et Alain Pagès, éd. B. H. Bakker et H. Mitterand, Montréal / Paris, Presses de l'Université de Montréal / Publications du CNRS, 1993.

17. Ibid., lettre du 28 mai 1899 , p. 505.

18. Ibid., lettre du 15 octobre 1898, p. 379.

19. Ibid., lettre du 5 mars 1899 , p. 409.

20. Ibid., lettre du 20 avril 1899, p. 461.

21. Ibid., lettre du 30 avril 1899.

22. Ibid., lettre du 4 novembre 1901, p. 729.

23. Jean-Christophe sera effectivement composé de dix volumes publiés de 1904 à 1912 dans les Cahiers de la Quinzaine. 
il importe de revenir, en amont, sur l'idéal très précoce, nourri dès 1886, d'un héros à la dimension cosmique, sur les projets de 1890, 1897 et 1901, enfin sur les fragments déjà écrits de 1896 à 190024. Ce que Bernard Duchatelet nomme la «préhistoire» de Jean-Christophe 25 permet de mesurer combien le roman-fleuve ressortit, plus encore qu'au projet, au rêve d'une conscience à peine sortie de l'adolescence (Romain Rolland a 20 ans en 1886), façonnée par la lecture de Tolstoï, l'écoute de la musique allemande et les promenades propices à l'illumination intérieure. L'idéal de l'écrivain, ses aspirations, ses goûts sont à la source même du héros Jean-Christophe Krafft, lequel accomplit le syncrétisme biographique de modèles comme Beethoven et Bach $^{26}$, mais est aussi un reflet de son jeune créateur. Plus précisément, Rolland décline le prisme de sa personnalité à travers le binôme formé par Jean-Christophe et Olivier, principales facettes d'un Moi pluriel, continuellement tiraillé entre l'Esprit et l'Action. Cette double orientation reste valable dans L'Âme enchantée (1922-1933), Annette trouvant dans sa sœur Sylvie le reflet inversé de ses propres instincts et s'évertuant, durant tout son parcours, à donner de la cohérence à un être multiple : «Est-ce qu['elle] n'en avait qu'une seule, âme? Elle en avait un troupeau, et, dans le tas, trois ou quatre de belle taille qui ne s'entendaient pas toujours ensemble $27 . . . »$ À bien considérer tout ce que l'auteur a placé de sa propre complexité dans ses personnages, le roman initiatique tend donc aussi, répétons-le, sur le mode asymptotique, vers l'écrit autobiographique pour lequel une prédilection évidente se manifestera dans Le Voyage intérieur (1942) 28 ou le Journal de Vézelay (1938-1944) récemment édité29. Le roman-fleuve peut aussi être lu comme le «songe d'une vie», songe fictif, allégorisé et musical, «ce rêve étrange et familier» fait par l'auteur au plus fort de son âme.

Pour faire saillir un paradigme davantage intime de l'écriture, il convient également de pousser l'investigation en aval des premiers chapitres de Jean-Christophe. Attardonsnous sur les infléchissements de nature autobiographique voulus de 1903 (avec la rédaction de L'Aube) à 1912 (avec la publication de La Nouvelle Journée qui vient clôturer le roman-cycle ${ }^{30}$ ) en notant d'abord le rôle majeur joué par la confidente Malwida von Meysenbug (1816-1903). En mars 1890, au cours d'une promenade sur le Janicule avec elle, le jeune Romain avait eu la «révélation » de ce que

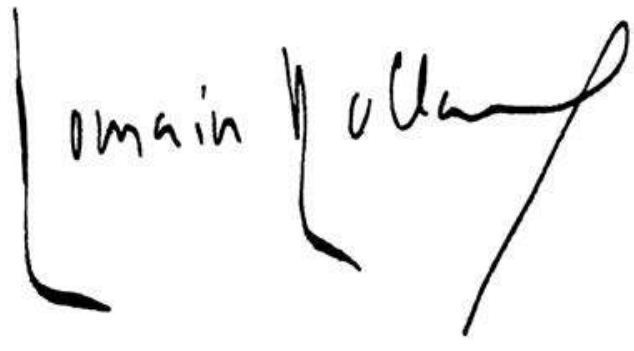

devrait être son futur héros ${ }^{31}$. En avril 1903, la vieille dame s'éteint en prononçant le mot «Liebe», Amour suprême qui inspire à l'auteur bouleversé l'idée de modifier la fin de son personnage ${ }^{32}$. Désormais, il s'agit d'orienter la mort de celui-ci du côté d'une compréhension annulant toute forme d'égoïsme et confinant à la fusion parfaite avec l'océan du monde 33 . Cette première évolution est primordiale, puisqu'elle fixe le contenu du graal spirituel sous-tendu dans la quête de Jean-Christophe. En outre, elle témoigne du fait que Rolland engage complètement les apprentissages de son vécu dans son travail de préparation et de rédaction. Tendance qui est confirmée fin 1904, alors que le troisième volume L'Adolescent est sur le point d'être publié. Cette fois-ci, la reprise des données autobiographiques est tellement évidente qu'elle donne à l'auteur la crainte d'en avoir trop écrit ${ }^{34}$. L'épisode d'Ada, la jeune femme avec qui Jean-Christophe entretient une liaison charnelle, n'est-elle

24. Bernard Duchatelet, Romain Rolland tel qu'en lui-même, Paris, Albin Michel, 2002, p. 122-123.

25. Bernard Duchatelet, La Genèse de Jean-Christophe de Romain Rolland, op. cit.

26. Les notes du voyage de sept semaines en Allemagne, effectué en 1896 avec Clotilde, constituent un précieux matériau de travail pour Rolland. 27. Romain Rolland, L’Âme enchantée, Paris, Albin Michel, 1967, p. 87. 28. Romain Rolland, Le Voyage intérieur. Songe d'une vie, édition augmentée, Paris, Albin Michel, 1959.

29. Romain Rolland, Journal de Vézelay. 1938-1944, éd. Jean Lacoste, Paris, Bartillat, 2012.

30. «Roman-cycle» et non pas «cycle», selon une précision à laquelle invite Christophe Pradeau (L'Idée de cycle romanesque : Balzac, Proust, Giono, thèse de doctorat, Paris VIII, 2000).

31. Romain Rolland, Mémoires et fragments du Journal, Paris, Albin Michel, 1946, p. 104.

32. Bernard Duchatelet, Romain Rolland tel qu'en lui-même, op. cit., p. 123.

33. Romain Rolland évoque «le sentiment océanique» dans une lettre à Sigmund Freud du 5 décembre 1927 (Un beau visage à tous sens. Choix de lettres de Romain Rolland (1866-1944), Paris, Albin Michel, 1967, p. 264-266).

34. Bernard Duchatelet, Romain Rolland tel qu'en lui-même, op. cit., p. 127. 
pas la transposition trop visible de la relation passionnée de l'écrivain avec sa première épouse Clotilde Bréal (18701946), dont il a divorcé en 1901 ? Quelques modifications sont effectuées au lieu du retrait de l'épisode un moment envisagé. Mais cette seule hésitation dans la publication dit assez que l'écrivain veut à la fois écrire une œuvre de pure fiction et alléger sa mémoire de souvenirs accablants, c'està-dire trouver l'impossible compromis entre la discrétion et l'indiscrétion. De même, il n'est pas anodin que l'un des volumes le plus difficile à achever soit La Révolte 35 , rédigé en Suisse durant l'été 1905 et dont le titre signale à lui seul la part de sédition qu'il contient au regard d'une trajectoire personnelle trop bien tracée. Peut-être le renoncement à une destinée confortable, mais subie, ce mouvement de mutinerie dans sa propre vie, est-il d'autant plus délicat à exposer qu'il est à certains égards la traduction d'une expérience personnelle complexe et récente. Transposition encore, en janvier-février 1910, avec Les Amies, huitième volume de la série dont le titre fait écho aux nombreuses femmes qui gravitent autour de l'auteur. Qu'on s'autorise un passage en revue pour comprendre combien ce volume est un vibrant hommage. Derrière les personnages se dessinent en effet une multitude de silhouettes aux contours plus ou moins nets : Malwida et Clotilde, on l'a dit, mais aussi Marie (18451919), la mère intransigeante, et Madeleine, la sœur cadette; la belle Sofia ${ }^{36}$ aimée dès 1890 et avec qui sont noués des liens d'une solide amitié; la comédienne Cora Laparcerie, modèle de Corinne; Louise Cruppi soutenue dans le deuil affreux de son fils; la sympathique, quoique trop empressée, Hélène Barrère; ou encore les admiratrices ${ }^{37}$ avec qui une relation épistolaire est parfois entretenue. Décidément oui, Les Amies est un hymne à la féminité et achève de nous convaincre du parti pris autobiographique de Rolland dans l'élaboration de son œuvre.

Concernant Jean-Christophe, une réserve peut toutefois être émise si l'on considère la part de plus en plus marquée, à partir de 1905, de la dénonciation des ambitions prussiennes et, plus globalement, de toute forme de bellicisme. Cette dénonciation pourrait tendre à faire passer l'intellectuel avant l'homme et à reléguer à l'arrière-plan les références qui font écho à la vie privée de l'auteur. Néanmoins, la disparition de celui-ci est loin d'être absolue. Tout se passe comme si les convictions du narrateur et celles du personnage reconduisaient encore et toujours à la conscience de l'écrivain.
Le paradigme de l'intrication implique un phénomène d'identification extrêmement marqué, qui n'est pas surprenant chez Rolland si l'on admet la parfaite cohérence d'une œuvre a priori seulement disparate. Il n'y a pas de solution de continuité entre les pièces de théâtre ${ }^{38}$, les biographies, les mémoires et, au milieu, la production romanesque qui coule comme un fleuve voué à irriguer une pensée s'efforçant de demeurer fidèle à elle-même. Quand il est question de Tolstoï, il est aussi question de Gandhi (la publication d'une lettre fait le lien dans la biographie de 191039) et quand il sera question de Gandhi, en 1924, «la réponse [formulée] dépasse[ra] infiniment $1^{\prime}$ Inde 40 », rejoignant in fine les thèses formulées dans Jean-Christophe, en même temps d'ailleurs que dans l'article «Au-dessus de la mêlée» de 191441. Lorsque Rolland s'empare de la vie d'un personnage - réel ou fictif, peu importe -, les rapports avec celui-ci sont fusionnels et conduisent à l'émergence d'une fraternité ignorant les frontières et le temps. Toujours, il s'agit de défendre, en empruntant sa symbolique à une poétique de la verticalité, les bienfaits d'une ascension au-dessus de la haine, contre les actions égoïstes qui fragmentent l'Être. Dans une symbiose idéale, l'individu est décentré de sa propre existence et conduit à s'élever pour embrasser tous les peuples, le cosmos et l'Amour lui-même qui régit cet oubli de soi. La réalité océanique est en lien direct avec le Rhin qui donne au roman-fleuve son assise métaphorique et que l'on retrouvait déjà en préambule de la Vie de Beethoven. Dans la biographie de 1903, Rolland l'évoque «si vivant, en effet, presque humain, pareil à une âme gigantesque où passent

\section{Ibid.}

36. Sofia Bertolini, née Guerrieri-Gonzaga.

37. Par exemple Clara van Ende, Agathe Lucardie ou Cosette Padoux. 38. Romain Rolland oscille, dans les années 1890, entre le projet d'un vaste roman et la rédaction de pièces de théâtre qu'il ne parviendra pas à faire jouer : Orsino, Empédocle, Les Baglioni, Niobé, Caligula, Le Siège de Mantoue et Jeanne de Piennes. La plupart de ses héros donnent à voir le Rêve en butte avec l'Action et problématisent la fusion idéale avec l'Éternel.

39. Romain Rolland, Vie de Tolstoï, préface de Stéphane Barsacq, Paris, Albin Michel, 2010, p. 233-236 : en 1913, une seconde édition permet d'ajouter des annexes, dont une lettre écrite par Tolstoï deux mois avant sa mort à Gandhi et datée du 7 septembre 1910.

40. Romain Rolland, Mahatma Gandhi, Paris, Stock, 1993 [1924], p. 130. 41. Romain Rolland, «Au-dessus de la mêlée», dans Au-dessus de la mêlée, Paris, P. Ollendorff, 1915. 
des pensées et des forces innombrables 42 ». La force nous propulse auprès de Jean-Christophe, selon une variation sur un même thème éminemment musicale, toujours semblable, jamais égale. Les genres, en l'occurrence biographique ou romanesque, offrent à l'auteur la possibilité de multiplier les modulations à l'envi et, sans jamais se répéter, de dire et redire sa confiance en une valeur suprême qui est le pacifisme.

En définitive, il apparaît que le modèle génétique de l'intrication invite à tisser de nombreux liens dans une œuvre extrêmement hétérogène, tandis que le paradigme du dépassement a pour corollaire la reconnaissance de territoires extrêmement variés et une volonté essentielle, manifeste chez Zola, de renouvellement. Cette distinction rejaillit sur le genre même du texte littéraire, avec d'un côté un «roman-fleuve» fondé sur la solidarité des volumes entre eux et la récurrence d'un héros fédérateur, de l'autre «un cycle romanesque» que vient nourrir l'exploration d'une multitude de thématiques. Cette opposition de deux conceptions de l'écriture mérite pourtant d'être nuancée, car elle n'est pas systématique et se dissout parfois dans la complémentarité.

\section{Complémentarité des deux paradigmes}

Un premier critère de différenciation réside dans la place accordée au Moi dans les avant-textes. Tandis que chez Zola, ces traces constituent une rareté, elles forment au contraire le nœud du projet rollandien, toujours en quête de nouvelles vies à raconter, comme autant de projections idéalisées. Or, ce que nous désignons comme paradigme de l'intrication peut malgré tout servir à éclairer le modèle zolien, dans la mesure où quelques concessions, justement significatives au regard de l'ensemble, sont faites au dépassement traditionnellement opéré. On ne peut en effet ignorer, dans les dossiers préparatoires, la prégnance d'un pronom personnel qui ne cesse de rappeler la part de l'auteur inscrite dans le projet d'écriture. On y voit «Je», «Nous», «On» tour à tour «pouvoir», «vouloir», « aimer», «avoir envie», «préférer», en somme assumer la part de subjectivité qui vient nécessairement dicter les grandes lignes du programme. Plus encore, dans la lignée de la formule jaillie dans le dossier du Rêve - «Moi, le travail, la littérature qui a mangé ma vie $[\ldots]^{43} \gg-$, on peut lire dans le dossier de Lourdes un raccourci de la philosophie zolienne : «Moi, toujours pour la libre nature ${ }^{44}$. $\gg$ Autrement dit, même si le sujet dépasse son vécu, il ne peut jamais se dépasser totalement lui-même, ni annuler sa présence dans les choix qu'il opère. Impossible objectivité du programme qui ne contrevient pas au projet naturaliste, mais contribue à en enrichir sa portée, la mythocritique de l'auteur participant de la dimension épique du roman en gestation.

Une deuxième variable des deux modèles génétiques se concentre sur le statut de l'universalité. La voici tantôt comprise dans le travail même de l'écrivain, comme abandon de soi au profit du cycle à écrire, tantôt dans la matière du roman-fleuve, aimanté par une vision océanique du monde. Ce rapport de l'Universel au contenant (le projet d'écriture zolien) ou au contenu (le Cosmos, horizon de Jean-Christophe et de L'Âme enchantée) peut toutefois être relativisé. Le Zola des Trois Villes, plus encore des Évangiles, est le chantre d'une religion laïque dont les vertus convergent vers celles énoncées dans les cycles rollandiens. En point de mire : la réconciliation des êtres avec le monde, leur acceptation de la Vie dans une compréhension totale et, malgré tous les malheurs qui surgissent, sereine, voire réjouie. Cette paix intérieure se trouve au terme du parcours imaginé par les deux auteurs pour des personnages aussi différents que Jean-Christophe, le docteur Pascal, Pierre Froment et ses enfants. Autant de signatures personnelles de deux écrivains aspirant à un équilibre bienfaiteur entre les forces humaines et celles d'une Nature accueillante.

Une dernière considération peut tenir lieu de conclusion. Finalement, ce qui permet le mieux de distinguer les deux paradigmes est sans doute la fonction accordée au sujet, institué tantôt conséquence, tantôt cause première de l'acte d'écriture. Le modèle du dépassement, pour sa part, est régi par la volonté de dire le monde au-delà de soi, mais reconduit tout de même Zola à lui-même dans la préférence accordée à tel dénouement, les clés de certains personnages, la tonalité accordée à tel roman, etc. C'est donc que le sujet découle

42. Romain Rolland, Vie de Beethoven, présentation de Jean Lacoste, Paris, Bartillat, 2015, p. 95.

43. Ébauche du Rêve, BnF, NAf 10322-10324, fos 221-222.

44. Deuxième Ébauche de Lourdes, BnF, MS NAF 1455, f ${ }^{\circ} 6$. 


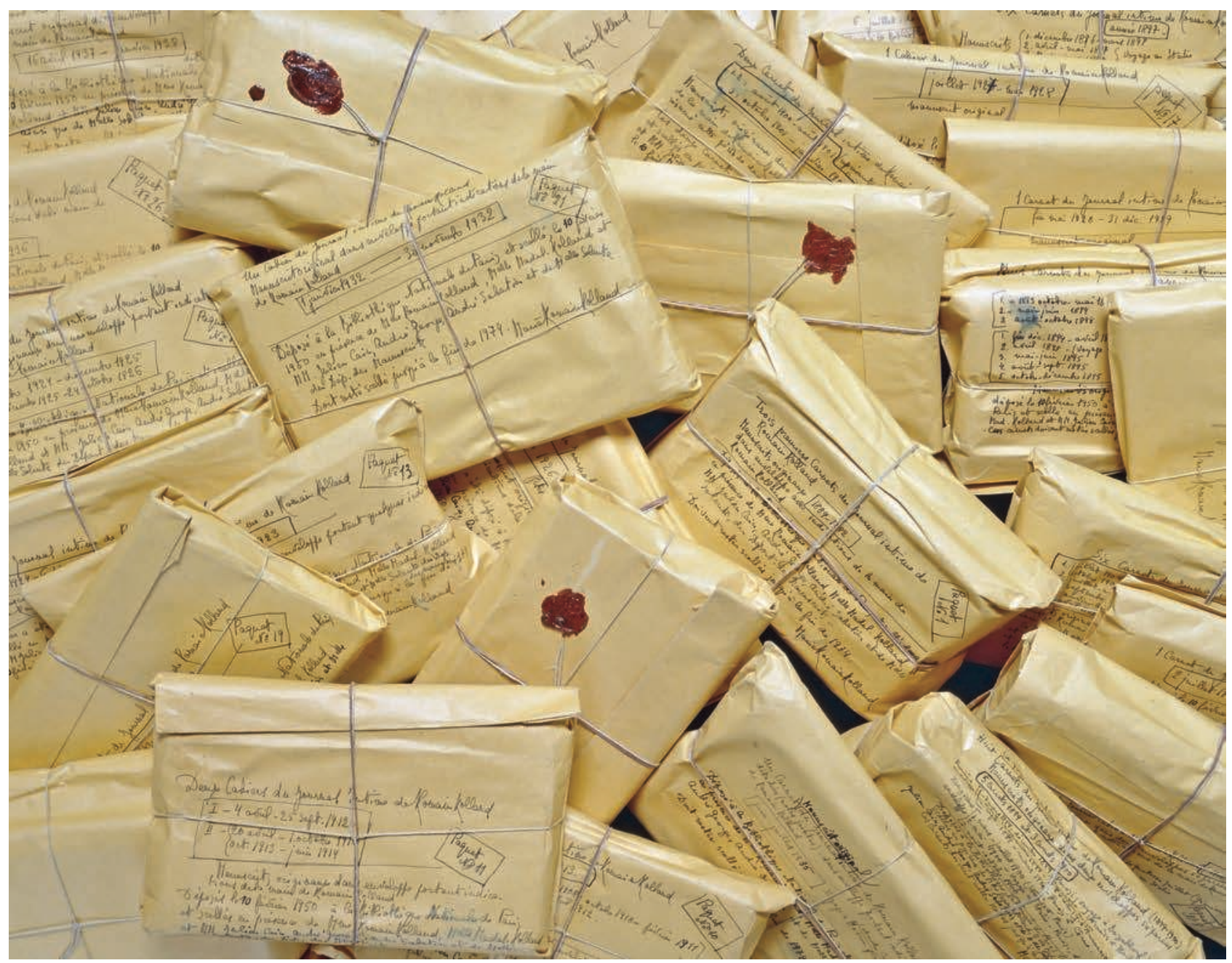

Fig. 1 : Paquets scellés du Journal de Romain Rolland légué par l'écrivain. Selon ses volontés, puis celles de sa veuve, Marie Romain Rolland, ils ne furent ouverts qu'en 2000 (Photo BnF / Département des Manuscrits)

de la genèse, en procède comme un appendice certes pas suffisant et très secondaire, mais nécessaire et inévitable. Au contraire, chez Rolland, l'instance auctoriale, qui modifie la trajectoire du roman-fleuve et influe directement sur le cheminement du personnage, est érigée en cause première de la dynamique génétique. La question reste donc de savoir si l'on peut résumer cette dissociation en posant un sujet ici subi et au maximum déconstruit, là choisi et sans cesse reconstruit. Il convient, une fois de plus, de nuancer la réponse et de penser davantage le dépassement et l'intrication comme deux manières complémentaires de mettre le sujet à distance sans jamais totalement le nier, en somme de le laisser être subsumé par une œuvre sublime et englobante. D'un côté, le sujet écrivant est capable d'une mainmise idéalement absolue sur l'avant-texte ainsi promu au rang de programme vis-à-vis de l'œuvre en gestation. Une telle posture implique un contrôle de soi quasi permanent et contribue à faire de l'auteur un chef d'orchestre aux pouvoirs démiurgiques. Le second modèle, en revanche, institue le sujet en objet d'écriture plus ou moins égotiste et tend à faire verser l'avant-texte dans le genre diariste. L'auteur accepte d'intégrer la partition de sa propre vie à la symphonie qu'il est en passe de composer, autrement dit instaure le lâcher-prise comme principe premier d'écriture et d'invention. Reste à savoir dans quelle mesure ces deux positions sont tributaires de l'appartenance ou non au mouvement naturaliste et quelles conséquences elles impliquent sur le plan stylistique. 
CÉLINE GRENAUd-Tostain est maître de conférences à l'université d'Évry-Val d'Essonne et membre de l'Équipe Zola de l'ITEM (CNRS-ENS). Ses travaux portent sur l'œuvre zolienne (édition critique de La Curée chez Classiques Garnier, collaboration à l'édition des Lettres à Alexandrine) et sur l'œuvre rollandienne (colloques «Tagore-Ray» en 2013 et «Centenaire d' "Au-dessus de la mêlée" » en 2014).

cgrenaud@yahoo.com

Les processus de l'écriture cyclique répondent à une double exigence et, ce faisant, à un paradoxe. D'un côté, l'orchestration générale de la symphonie romanesque, nécessairement pensée en amont, doit garantir la cohérence de l'ensemble. Mais de l'autre, l'écrivain s'octroie d'emblée la liberté de déverrouiller, si nécessaire, les portes bloquées par son texte programmatif. Or, la résolution de ce paradoxe trouve, dans l'œuvre de Zola et celle de Rolland, deux modèles représentatifs et complémentaires. Il s'agit de mesurer combien la dimension autobiographique occupe une place déterminante dans la construction des cycles rollandiens, tandis qu'elle est largement supplantée, dans la genèse des cycles zoliens, par le souci, évidemment naturaliste, de faire coïncider l'investigation sur le terrain au «saut dans les étoiles» voué à propulser l'œuvre dans l'épopée ou le mythe.

Cyclic writing processes meet a double demand and by doing so, lead to a paradox. On the one hand, the overall orchestration of a fictional symphony, necessarily thought out in advance, must guarantee the whole's coherence. But on the other, the writer grants himself the freedom to unlock if necessary the doors blocked by his programmatic text. Now in Zola and Rolland's works there are two representative and complementary models for resolving this paradox. The question is to measure to what degree the autobiographic dimension is decisive in the construction of Rolland's cycles, while in Zola's cycles it is largely replaced by the Naturalist desire to make the fieldwork coincide with the "leap to the stars" bound to propel the work into the epic poem or myth.

Die Prozesse des zyklischen Schreibens entsprechen einem zweifachen Bedürfnis und zugleich einem Paradox. Einerseits muss die allgemeine Orchestrierung der Romansymphonie, die notwendigerweise schon im Entwurf vorhanden ist, die Kohärenz des Ganzen sicherstellen. Aber andererseits nimmt sich der Autor die Freiheit, wenn nötig, jene Tore zu entriegeln, die durch seinen Entwurf zunächst verschlossen sind. Die Auflösung dieses Paradoxes führt im Werk von Zola und in jenem von Rolland zu zwei repräsentativen und komplementären Modellen. Es geht darum, festzustellen, wie bestimmend die autobiographische Dimension in der Konstruktion der Zyklen Rollands ist, während sie in den Zyklen Zolas weitgehend verdrängt wird durch das naturalistische Bestreben, die Quellenstudien vor Ort mit dem „Sprung zu den Sternen“ zusammenfallen zu lassen, sodass das Werk ins Epische oder ins Mythische mündet.

\section{Zola et Rolland : deux paradigmes du travail génétique}

Los procesos de escritura cíclica corresponden a una doble exigencia y, consecuentemente, a una paradoja. Por un lado, la orquestación general de la sinfonía novelesca, necesariamente preconcebida, debe garantizar la coherencia del conjunto; pero, por el otro, el escritor se otorga desde el comienzo la libertad de desbloquear, en caso de necesidad, las puertas trabadas por su texto programático. La resolución de esta paradoja encuentra, en la obra de Zola y en la de Rolland, dos modelos representativos y complementarios. Intentaremos evaluar hasta que punto la dimensión autobiográfica ocupa un sitio determinante en la construcción de los ciclos de Rolland, mientras que en la génesis de los ciclos de Zola, esta dimensión es reemplazada por la voluntad, puramente naturalista, de hacer coincidir las investigaciones de terreno con el "salto a las estrellas" destinado a inscribir la obra en la epopeya o el mito.

Os processos de escrita cíclica respondem a uma dupla exigência e, por aí, a um paradoxo. Por um lado, a orquestração geral da sinfonia romanesca, necessariamente pensada a montante, deve garantir a coerência do todo. Mas, por outro lado, o escritor não abdica do direito de abrir novas vias que, no seu programa de conjunto, pareciam cerradas. A resolução deste paradoxo encontra, na obra de Zola e na de Rolland, dois modelos representativos e complementares. A dimensão autobiográfica ocupa um papel determinante na construção dos ciclos rolandianos, o que é largamente suplantado, na génese dos ciclos zolianos, pela preocupação, obviamente naturalista, de fazer coincidir a pesquisa no terreno com o "salto para os astros" que confere à obra dimensões de epopeia ou de mito.

I procedimenti della scrittura ciclica rispondono a una doppia esigenza e perciò a un paradosso. Da un lato, l'orchestrazione generale della sinfonia romanzesca, necessariamente pensata a monte, deve garantire la coerenza dell'insieme; dall'altro, lo scrittore si concede subito la libertà di aprire, se necessario, le porte bloccate dal suo testo programmatico. La soluzione di questo paradosso trova, nell'opera di Zola e in quella di Rolland, due modelli rappresentativi e complementari. Si tratta di misurare quanto la dimensione autobiografica occupa un posto determinante nella costruzione dei cicli di Rolland, mentre è decisamente soppiantata, nella genesi dei cicli zoliani, dalla preoccupazione - chiaramente naturalista - di far coincidere l'investigazione sul campo al "salto nelle stelle", destinato a proiettare l'opera nell'epopea o nel mito. 